\title{
Roma-BZCAT: a multifrequency catalogue of blazars
}

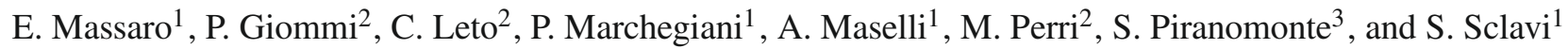 \\ 1 Dipartimento di Fisica, Sapienza Università di Roma, Piazzale A. Moro 2, 00185, Roma, Italy \\ e-mail: enrico.massaro@uniroma1.it \\ 2 ASI Science Data Center, ASDC c/o ESRIN, via G. Galilei, 00044 Frascati, Italy \\ 3 INAF, Osservatorio Astronomico di Roma, Monte Porzio Catone, Italy
}

Received 8 May 2008 / Accepted 4 September 2008

\begin{abstract}
We present a new catalogue of blazars based on multifrequency surveys and on an extensive review of the literature. Blazars are classified as BL Lacertae objects, as flat spectrum radio quasars or as blazars of uncertain/transitional type. Each object is identified by a root name, coded as BZB, BZQ and BZU for these three subclasses respectively, and by its coordinates. This catalogue is being built as a tool useful for the identification of the extragalactic sources that will be detected by present and future experiments for $\mathrm{X}$ and gamma-ray astronomy, like Swift, AGILE, Fermi-GLAST and Simbol-X. An electronic version is available from the ASI Science Data Center web site at http://www.asdc.asi.it/bzcat.
\end{abstract}

Key words. BL Lacertae objects: general - galaxies: quasars: general - catalogs

\section{Introduction}

Blazars are a relatively rare class of active galactic nuclei (AGN) characterised by an electromagnetic emission over the entire energy spectrum, from the radio band to the most energetic $\gamma$ rays. Angel \& Stockman (1980) reported that the word BLAZAR, a combination of BL Lac object and quasar, was proposed by Edward A. Spiegel in a talk given at the banquet of the famous first BL Lac Conference in Pittsburgh (1978). At that epoch these sources were compact flat-spectrum radio sources with a violent flaring activity, featureless optical spectra and occasionally showing a high linear polarisation, as first recognised by Strittmatter et al. (1972). Their emission in the X and $\gamma$-ray bands was practically unknown. This name was lucky because it was soon adopted and became official with the review paper by Angel \& Stockman (1980). These authors gave also one of the first lists of BL Lac objects containing 57 sources.

The discovery that some radio-loud quasars show characteristics similar to BL Lac objects, with the exception of broad spectral lines, was important for a complete definition of the blazar properties. Moore \& Stockman (1981) gave an important contribution when performed a polarisation survey in which discovered 17 high polarisation quasars (HPQ) and discussed their relation with BL Lacs. Before their work only four radio quasars were known to show high variable optical linear polarisation. Some years later Impey \& Tapia (1988) extended the polarisation surveys and discovered 31 new blazars.

The unusual properties of blazars were explained at the Pittsburgh Conference by Blandford \& Rees (1978) in terms of non-thermal emission from small regions moving down a jet with a velocity close to the speed of light and observed at a small angle with respect to the line of sight. The Doppler boosting that results from these very special circumstances strongly amplifies the non-thermal emission that dominates with respect to all other components of thermal origin, like those from the accretion disk, the dust heated by the AGN flux, or from stars and interstellar matter of the host galaxy.

Many surveys have been carried out to identify blazars and their number is continuously increasing. Nevertheless, only a relative small number of blazars have been studied intensively. There are still many unsolved problems about the origin of the blazar phenomenon, the physical processes occurring in the nuclear region, and about the cosmological evolution of these sources. Furthermore, because of their very broad spectral emission, blazars are expected to play an important role in generating the extragalactic background and its fluctuations in several energy bands.

Blazars constitute the most numerous class of extragalactic $\gamma$-ray sources detected by the EGRET experiment onboard Compton-GRO (Hartman et al. 1999), and by atmospheric Cerenkov telescopes at $\mathrm{TeV}$ energies. Moreover, a recent analysis of the arrival directions of cosmic rays with energy above $\sim 6 \times 10^{19} \mathrm{eV}$ collected at the Auger observatory, showed a significant correlation with the coordinates of AGNs within $\sim 75 \mathrm{Mpc}$ (Pierre Auger Collaboration 2007). The knowledge of the blazar population is, therefore, very relevant in high-energy astrophysics.

For these reasons a complete list of known blazars, based on an accurate examination of literature data, can be very useful for carrying out some of the scientific goals of present and future space observatories like Fermi-GLAST, Planck, and Simbol$\mathrm{X}$. In this paper we describe the "Multifrequency Catalogue of BLAZARS", also named Roma-BZCAT, that will be published in four volumes (the first two are already available; Massaro et al. 2005, 2008) each of them containing a sky region covering $6 \mathrm{~h}$ of right ascension. This catalogue is a master list of sources useful for the search of counterparts in different spectral bands and for the selection of samples for statistical studies of blazar properties and evolution. An electronic version is also available on-line from the ASDC web 
page (http: //wWw . asdc.asi.it/bzcat), where it is updated as new objects are reported.

\section{Blazar surveys}

Starting from the late 1980's, several sizable blazar samples were produced as a result of a number of systematic searches in large radio or X-ray surveys. These highly organised efforts were initially carried out following a standard approach involving the optical identification of all sources above the survey's flux limit. However, when the need to assemble deeper and larger samples pushed the need for optical telescope time to hardly manageable amounts, multi-frequency (usually radio, optical, and X-ray), pre-selection techniques were developed in order to significantly reduce the number of candidates requiring optical spectroscopy.

After the first list of 32 BL Lac objects given by Stein et al. (1976), Angel \& Stockman (1980) increased the number to 57. A catalogue of BL Lac objects was prepared by Burbidge \& Hewitt (1987), containing 87 sources, but it did not include all the sources listed by Angel and Stockman. At that epoch the total number of blazars was 103. Since 1984 Veron-Cetty and Veron published a general catalogue of AGNs that, starting from the 2nd edition (Veron-Cetty \& Veron 1985), included a table of BL Lac objects, whose number is continuously increasing from the original 73 to 1122 of the 12th edition (Veron-Cetty \& Veron 2006). Although very useful, the VCV catalogue is not a blazar catalogue, because many sources are listed in the BL Lac table and others among the quasars, and for this reason, the blazar nature of the sources is not directly apparent.

A sample oriented catalogue was compiled by Padovani \& Giommi (1995) including all BL Lacertae objects that were known at that time. After ten years that list has become largely incomplete and needs updating.

In recent years new surveys have become available and the number of blazar candidates has grown rapidly. An important multifrequency sample is the radio-optical-X-ray built at ASDC (ROXA, Turriziani et al. 2007), which was obtained by means of a cross-correlation between large radio (NVSS, ATCAPMN Taker 2000) and X-ray surveys (RASS), together with the SDSS-DR4 and $2 d F$ data to spectroscopically identify the candidates. The ROXA sample consists of 816 objects and includes 173 newly discovered blazars. All the ROXA objects were examined to verify that they satisfy the Roma-BZCAT acceptance criteria, and only a small percentage of them was not accepted. The relatively high threshold of the X-ray flux of the RASS survey mainly selects objects with high $F_{\mathrm{X}} / F_{\text {radio }}$ flux ratio, hence HBL objects.

Another sample of BL Lac candidates, including 386 sources, was obtained by Collinge et al. (2005) using the SDSS spectroscopic database in a field of $2860 \mathrm{deg}^{2}$. These sources were divided into the two groups of 240 "probable" and 146 "possible" BL Lac candidates, the former selected by colours, unlike those of DC white dwarf stars, and, some evidence of extragalactic nature, such as a redshift estimate and/or a radio/X-ray counterpart. The majority of these BL Lac candidates are, as for the ROXA sample, of the HBL type. In this sample there are several sources without a radio counterpart, and this possibility raises the issue of the existence of radioquiet (or optically selected) BL Lacs. We are already faced with this problem when considering the sample of "optically selected" BL Lac candidates by Londish et al. (2002), as a byproduct of the much wider $2 Q Z$ sample of QSOs (Croom et al. 2001). Nesci et al. (2005) searched for optical variability of a subsample of the $2 Q Z-B L$ sources and found that only a few of them have significant brightness changes, including all the radio-loud ones. In a recent paper, Londish et al. (2007) reported a further analysis of an expanded and revised sample of potential optically identified BL Lacs and concluded that there can be no significant population of radio-quiet BL Lac objects.

Although in principle we cannot exclude the existence of high- $z$ HBL objects with a sub mJy flux density in the GHz band, we preferred to maintain our criterion to include in the RomaBZCAT only those sources with a confirmed or high confidence radio counterpart; however, in Table 2 of the 12th edition of their AGN catalogue (Véron-Cetty \& Véron 2006) reported all the BL Lac candidates given in the above samples, therefore including also radio-quiet objects, but clearly indicating that they are not confirmed.

A new important survey aimed at identifying new blazars is the Candidate Gamma Ray Blazar Survey (CGRaBS) (Healey et al. 2008). This sample is essentially based on the CRATES (Combined Radio All-Sky Targeted Eight GHz Sources) catalogue (Healey et al. 2007), which extended the CLASS (Cosmic Lens All-Sky Survey, Myers et al. 2003) to obtain observations at $8.4 \mathrm{GHz}$ of flat spectrum sources with $|b|>10^{\circ}$ and brighter than $65 \mathrm{mJy}$ at $4.8 \mathrm{GHz}$, with the exclusion of the region with declination $\delta>75^{\circ}$ where the flux limit is $250 \mathrm{mJy}$. The CRATES catalogue contains 11131 sources, many of them without an optical counterpart.

The CGRaBS sample was selected to identify sources with the greatest similarity to the EGRET blazars, and an optical follow-up provided the counterpart identification and spectroscopy for the majority of them. For the sake of completeness, we decided to add all $C G R a B S$ sources classified as FSRQs to the Roma-BZCAT, although their spectra are not available in the literature and/or on-line. These sources are marked by the note CG. Sources of unknown type and without redshift were not included.

Table 1 gives a list of all the major surveys of both types that led to the discovery of most of the blazars known today and included in this catalogue.

\section{Criteria for source selection}

As stated in the introduction, blazars come in two main flavours: BL Lac objects (BL) and Flat Spectrum Radio Quasars (FSRQ). The former type is characterised by featureless optical spectra, while those of FSRQs display the prominent emission lines that are typical of QSOs. This classification, although very simple, is not unambiguous. For instance, well-established BL Lac objects like OJ 287 and BL Lac itself on some occasions clearly exhibited emission lines, indicating common nature of the two types of blazars. Another problem concerns variability: sometimes blazars showed large brightness changes on time scales of a day or of a few hours, whereas it is possible that they remain in a quasi-quiescent state for years. Such a behaviour can hide blazar properties and can lead to misidentification. In very general terms we can define a blazar as an extragalactic source characterised by $i$ ) a spectral energy distribution (SED) showing strong non thermal emission over the entire electromagnetic spectrum, from radio waves to $\gamma$-rays; and ii) the presence of some evidence for relativistic beaming. It is clear that both requirements can be secured by only making use of rich observational data sets, involving either polarimetric or variability studies and VLBI imaging to detect compact cores and superluminal motions. These data sets are available only for a limited number of, usually bright, sources, and therefore the majority of known blazars have been recognized only on the basis of a few spectral properties. Moreover, the data available are not simultaneous 
Table 1. Major blazar surveys.

\begin{tabular}{|c|c|c|c|c|}
\hline Survey name & Blazar type & Survey type & $\begin{array}{c}\begin{array}{c}\text { Number } \\
\text { of objects }\end{array} \\
\end{array}$ & "Reference \\
\hline 2 Jansky & BL Lacs+FSRQs & $\begin{array}{l}\text { radio flux limited } \\
F(2.7 \mathrm{GHz})>2 \mathrm{Jy}, \alpha_{r} \leq 0.4\end{array}$ & 60 & $1,2,3$ \\
\hline 1 Jansky & BL Lacs & $\begin{array}{l}\text { radio flux limited } \\
F(5 \mathrm{GHz})>1 \mathrm{Jy}, \alpha_{r} \leq 0.5\end{array}$ & 34 & 4 \\
\hline 1 Jy NVSS-RASS & BL Lacs+FSRQs & $\begin{array}{l}\text { radio, opt, } X \text {-ray selection } \\
\text { radio fl. limited, } F(1.4 \mathrm{GHz})>1 \mathrm{Jy}\end{array}$ & 157 & 5 \\
\hline 1 Jy WMAP & BL Lacs+FSRQs & $\begin{array}{l}\text { microwave flux limited } \\
F(61 \mathrm{GHz}) \gtrsim 1 \mathrm{Jy}\end{array}$ & 180 & 6,7 \\
\hline Parkes $0.25 \mathrm{Jy}$ & BL Lacs+FSRQs & $\begin{array}{l}\text { radio flux limited } \\
F(2.7 \mathrm{GHz})>0.25 \mathrm{Jy}\end{array}$ & 371 & 8,9 \\
\hline EMSS & BL Lacs & $\begin{array}{l}\text { X-ray flux limited } \\
F_{X} \gtrsim 2 \times 10^{-13} \mathrm{erg} \mathrm{cm}^{-2} \mathrm{~s}^{-1}\end{array}$ & 41 & 10,11 \\
\hline IPC slew survey & BL Lacs & $\begin{array}{l}\text { X-ray flux limited } \\
F_{X} \gtrsim 2 \times 10^{-12} \mathrm{erg} \mathrm{cm}^{-2} \mathrm{~s}^{-1}\end{array}$ & 51 & 12 \\
\hline DXRBS & BL Lacs+FSRQs & $\begin{array}{l}\text { radio+opt }+ \text { X-ray selection } \\
\text { radio fl. limited } \\
F(5 \mathrm{GHz})>50 \mathrm{mJy}\end{array}$ & 227 & 13,14 \\
\hline REX & BL Lacs & radio $+o p t+X$-ray selection & $\sim 100$ & 15,16 \\
\hline RGB & BL Lacs & radio+opt+X-ray selection & 127 & 17 \\
\hline Sedentary & extreme BL Lacs & $\begin{array}{l}\text { radio }+ \text { opt }+ \text { X-ray selection } \\
\text { radio } \mathrm{fl} \text {. limited } \\
F(1.4 \mathrm{GHz})>3.5 \mathrm{mJy}\end{array}$ & 150 & 18,19 \\
\hline CLASS & BL Lacs+FSRQs & $\begin{array}{l}\text { radio+opt } \\
F(5 \mathrm{GHz})>30 \mathrm{mJy}, \alpha_{r} \leq 0.5\end{array}$ & 147 & 20,21 \\
\hline Gamma-ray Blazars & BL Lacs+FSRQs & radio $+\mathrm{X}$-ray selection & 241 & 22 \\
\hline 2dF BL Lac Survey & BL Lacs & Optical selection & 56 candidates & 23,24 \\
\hline SDSS BL Lac Survey & BL Lacs & Optical selection & 347 & 25 \\
\hline ROXA & BL Lacs+FSRQs+AGN & radio $+o p t+X-$ ray selection & 816 & 26 \\
\hline CGRaBS & BL Lacs+FSRQs+AGN & radio flux limited & 1625 & 27 \\
\hline SDSS-FIRST BL Lac Survey & BL Lacs & Optical+radio selection & 501 & 28 \\
\hline
\end{tabular}

(1) Wall and Peacock (1985), (2) di Serego-Alighieri et al. (1994), (3) Urry, Padovani (1995), (4) Stickel et al. (1991), (5) Giommi et al. (2002), (6) Bennett C.L. et al. (2003) (7) Giommi, Colafrancesco, (2004), (8) jackson et al. (2002), (9) Wall et al. (2005), (10) Stocke et al. (1991), (11) Rector et al. (2000), (12) Perlman et al. (1996), (13) Perlman et al. (1998), (14) Landt et al. (2001), (15) Caccianiga et al. (1999), (16) Caccianiga et al. (2002a), (17) Laurent-Muehleisen et al. (1998), (18) Giommi, Menna, Padovani (1999), (19) Giommi et al. (2005), (20) Marchã et al. (2001), (21) Caccianiga et al. (2002b), (22) Sowards-Emmerd et al. (2005), (23) Londish et al. (2002), (24) Londish et al. (2007), (25) Collinge et al. (2005), (26) Turriziani et al. (2007), (27) Healey et al. (2008), (28) Plotkin et al. (2008).

in most cases and are often derived from catalogues, sometimes making it very difficult to establish whether an AGN is a genuine blazar or not.

For these reasons we adopted a rather wide set of acceptance criteria as described below:

1. detection in the radio band, down to mJy flux densities at $1.4 \mathrm{GHz}$ (NVSS, Condon et al. 1998 or FIRST; White et al. 1997) or $0.84 \mathrm{GHz}$ (SUMSS, Mauch et al. 2003);

2. optical identification and knowledge of the optical spectrum to establish the type of blazar;

3. isotropic X-ray luminosity close to or higher than $10^{43} \mathrm{erg} \mathrm{s}^{-1}$

4. for FSRQs a spectral index $\left(F(v) \propto v^{-\alpha}\right)$, measured between $1.4 \mathrm{GHz}$ (or $0.843 \mathrm{GHz}$ ) and $5 \mathrm{GHz}, \alpha_{\mathrm{r}}<0.5$, but not for BLs, although most of them have flat spectra;

5. compact radio morphology, or, when extended, with one dominant core and a one-sided jet.

Recent observational campaigns with the Swift-XRT telescope on samples of BL Lacs and FSRQs without measured X-ray fluxes confirmed that they emit $\mathrm{X}$ rays with an intensity in agreement with that of similar blazars (Giommi et al. 2007).

Some authors have proposed lists of optically selected BL Lacs on the basis of featureless spectra without the occurrence of a radio and X-ray counterpart (e.g. 2dF BL Lac survey).
These sources are reported in some widely used catalogues, although with a doubtful indication. To reduce the possibility of including non blazar sources in the catalogue, we adopted a conservative criterion and inserted only those sources in the table of candidate BL Lacs having well-established radio emission.

Another problem concerns the nature of a class of radio sources whose nature is still a matter of debate: the so called High-Frequency Peakers (HFP) (Dallacasa et al. 2000) and GHz-Peaked Sources (GPS) (O'Dea et al. 1991), whose optical counterparts are a mixture of galaxies and quasars, together with a few BL Lacs. Broad-band monitoring campaigns have shown that some of these sources are indeed variable and that their spectra can change from the characteristic convex shape to a flat frequency distribution (Torniainen et al. 2005). Such behaviour suggests a link between GPS-HFP objects and blazars. Moreover, the spectral coverage of many radio sources is poor, both in frequency and time, and several sources with non simultaneous spectra could have been classified as FSRQ, while they are GPS-HFP and vice-versa. In other cases, some SEDs indicate the occurrence of a convex radio component superposed onto a power-law spectrum. We decided therefore to include this type of radio sources in the catalogue, having blazar characteristics and adding a specific note if they are GPS or HFP, using the recent master list by Labiano et al. (2007) and other literature as reference. 


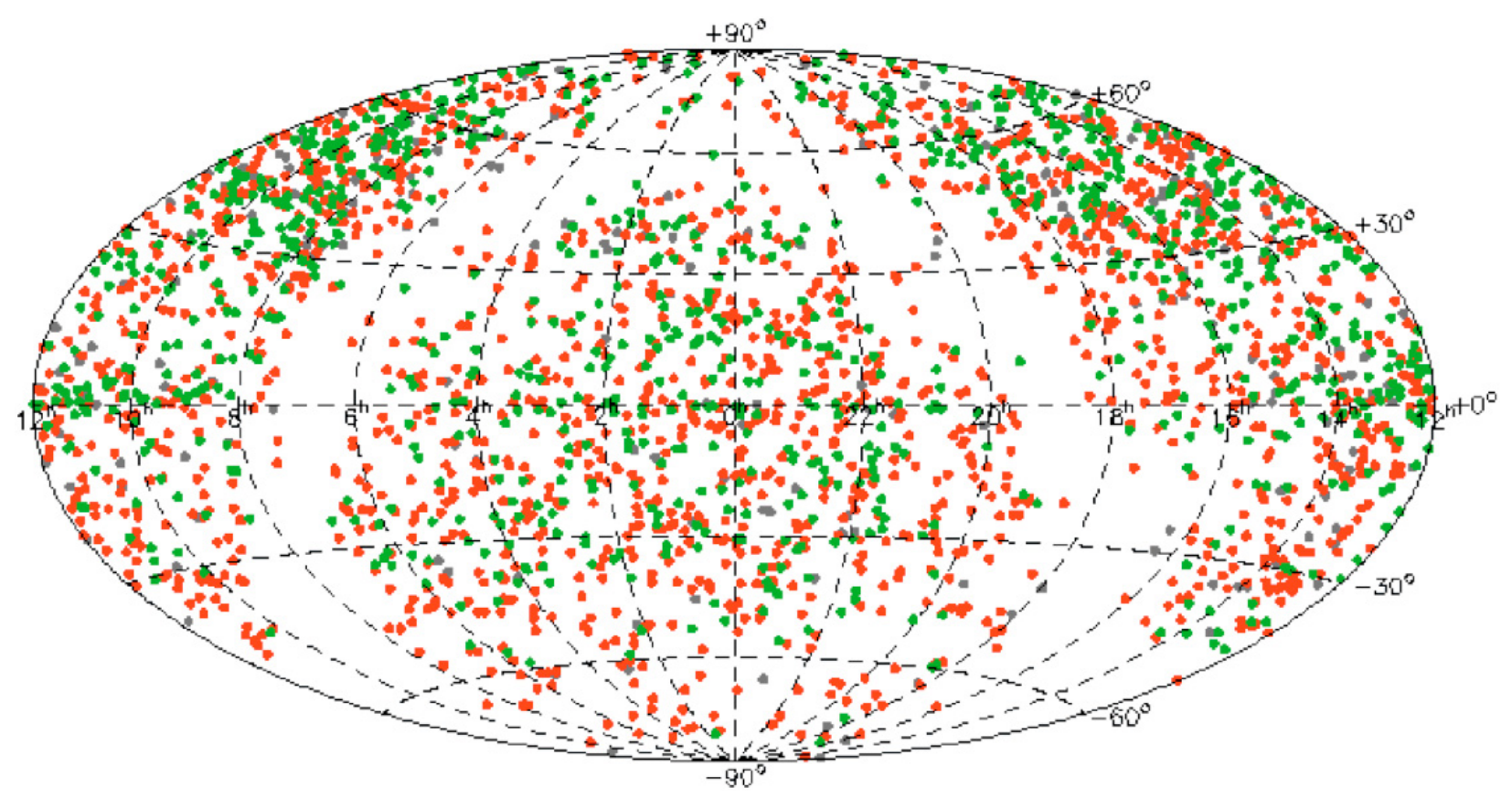

Fig. 1. Hammer-Aitoff projection in equatorial coordinates showing the sky distribution of blazars listed in the on-line version of Roma-BZCAT. Red points indicate BZB sources, green points BZQ objects, and blue points BZU sources; the empty belt corresponds to the Milky Way and the most crowded region in the northern emisphere to the SDSS field.

\section{General organisation of the Roma-BZCAT}

The Roma-BZCAT is structured in two parts. Part 1 , at present the only one available on-line, contains the lists of blazars, which are classified in three main groups, according to their spectral properties. Each blazar is identified by a three-letter code, where the first two are $\mathbf{B Z}$ for blazar and the third one specifies the type, followed by the truncated equatorial coordinates (J2000).

The codes are:

- BZB: BL Lac objects, used for AGNs with a featureless optical spectrum or only with absorption lines of galaxian origin and weak and narrow emission lines;

- BZQ: flat-spectrum radio quasars, with an optical spectrum showing broad emission lines and dominant blazar characteristics;

- BZU: blazars of uncertain type, adopted for sources with peculiar characteristics but also showing blazar activity: for instance, occasional presence/absence of broad spectral lines or features, transition objects between a radio galaxy and a BL Lac, galaxies hosting a low luminosity blazar nucleus, etc.;

The BZB objects are listed in the first two tables of the catalogue (but they are not distinguished in the on-line version). Table I contains all firmly identified BL Lacs, whereas Table II includes sources considered to be candidate BL Lac objects, in particular those for which we could not find, either in literature or on the web, an optical spectrum or a good description. The BZQ sources listed in Table 3 are FSRQs and other quasars showing evident blazar characteristics like a strong and fast optical variability (OVV sources) and a high linear polarisation (HPQ sources). Finally, we listed in Table 4 the BZU sources with a short note explaining the reason for this classification.

The following data are reported for each source: J2000 coordinates, $R$ magnitude (from USNO-B1), radio flux density at $1.4 \mathrm{GHz}$ (from NVSS or other catalogues, if not known the flux at another frequency is given), the X-ray flux in the $0.1-2.4 \mathrm{keV}$ energy interval, mostly from ROSAT archive, and the redshift. Some useful synthetic notes are also given for a limited number of blazars: e.g. member of a galaxy cluster, detection of emission lines, etc.

The on-line version reports the same data and offers the additional possibility of using the ASDC "Data Explorer" tool. This tool is being developed at the ASDC to help search for more information from several on-line databases. In particular, for each source, it provides a direct link to NED and SIMBAD, which can be used to find other names, references, and other useful information.

\section{Blazar population and spectral energy distributions}

The total number of blazars presently listed in the Roma-BZCAT is 2728 and their distribution in the sky is shown in Fig. 1. The most populated class is that of FSRQs, which are $57.1 \%$ of sources. BL Lac objects (including candidates) are $33.9 \%$, and the remaining $9.0 \%$ are sources of uncertain classification. Only about the $42 \%$ of FSRQs are detected in the X-ray band. A large observational work is then necessary to complete the knowledge of the emission from FSRQs in this band.

A relevant and well known difference between the populations of FSRQs and BL objects is the redshift distributions as shown by the histograms given in Fig. 2. BL Lacs are much closer than FSRQs and their distribution peaks at $z \simeq 0.3$, whereas quasars show a broad maximum between 0.6 and 1.5. There are only very few BL objects at redshift higher than 0.8 , but these estimates are generally very uncertain, and the extension of the distribution in this range cannot be considered fully reliable. One must also take into account that $\mathrm{z}$ is unknown for about $40 \%$ of BZB sources and that there is a bias towards near and low power sources whose galaxian lines are easily detectable. 


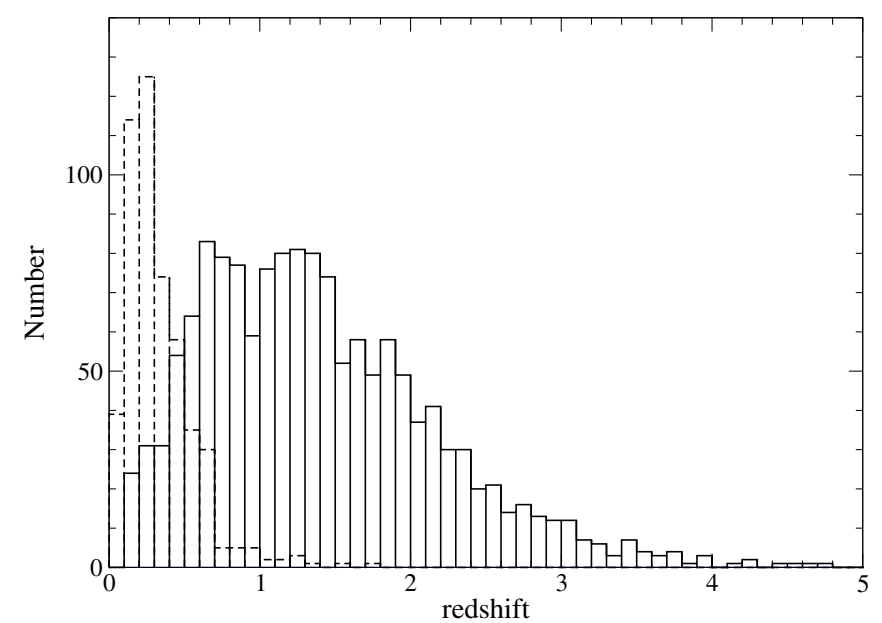

Fig. 2. The redshift distributions of BL Lac objects, including candidate sources (dashed histogram) and of FSRQs (solid line histogram) reported in the Roma-BZCAT.

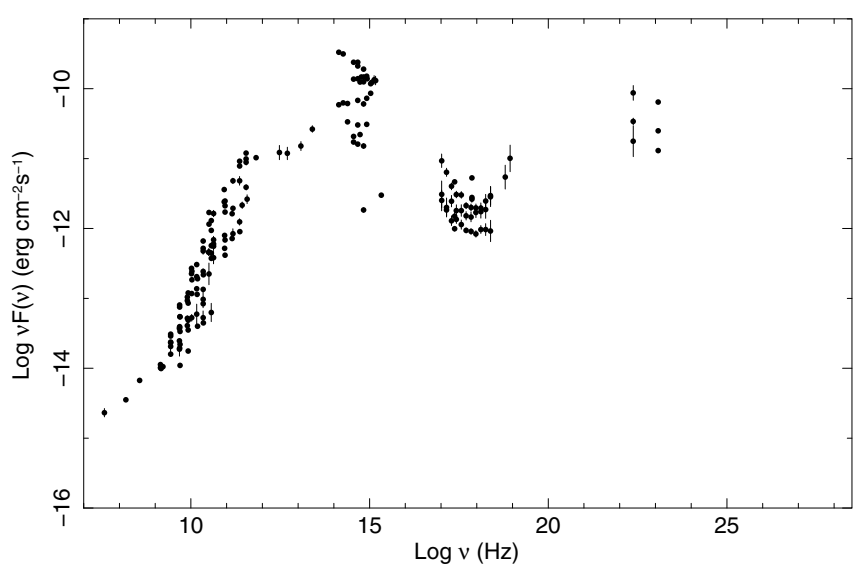

Fig. 3. The SED of the BL Lac object BZB J0721+7210 (S5 0716+714) derived from the Roma-BZCAT database.

A section of the Roma-BZCAT is devoted to presenting detailed data and other useful information for a selection of interesting blazars to represent the various blazar types and with special attention to those detected in the $\gamma$ - and hard X-ray ranges. In particular, for each object we build the SED using a large collection of non-simultaneous multi-frequency data. Although SEDs of extragalactic sources are indeed given in the NED database, we made an effort to enrich fluxes derived from catalogues by adding more data taken from papers and observational databases. In the case of monitoring campaigns containing a lot of data we considered only the lowest and highest flux levels. An example, the SED of the well known BZB J0721+7120 (S5 0716+714, 3EG J0721+7120), is shown in Fig. 3.

\section{Conclusion}

The main scientific goals that led us to the compilation of the Roma-BZCAT were

i) To have the most complete list of published blazars, useful for identifying the counterparts of high-energy sources (in this respect the Roma-Bzcat is already one of the reference catalogues of the Fermi-GLAST mission).

ii) To have a list of extragalactic objects useful for studying the populations of high-energy extragalactic sources. iii) To have a population from which it will be possible to extract samples satisfying statistical criteria for investigating blazar properties and evolution.

iv) To have a large database of SEDs for different types of blazars useful for studying radiation mechanisms and relativistic beaming effects.

An open problem is that of the completeness of the catalogue. There is, in fact, a deficiency of blazars in the southern sky because of the fewer surveys than the northern emisphere. Many new discovered blazars and candidates, for instance, have been identified using spectroscopic observations available in the $S D S S$. A similar research project for exploring the region around the southern celestial pole, possibly coupled with a high sensitivity radio survey, will certainly lead to the discovery of hundreds of new blazars. Moreover, we expect that the deeper search for faint optical counterparts of flat spectrum radio sources will surely increase the number of blazars at high redshift and provide new useful information for understanding their cosmological evolution. To take such new possible developments into account, we will continue in the next years to update the on-line version of the Roma-BZCAT and to revise the data and, eventually, the classification of sources.

Acknowledgements. This work has made extensive use of the following on-line facilities: the National Extragalactic Database (NED), The Astrophysics Data System (ADS), SIMBAD, NRAO calibrator database, ASDC. We acknowledge the financial support by Agenzia Spaziale Italiana (ASI-INAF n. I/010/06/0) for astrophysical science activity for the Fermi-GLAST mission. We thank Roberto Primavera for his help in the preparation of the on- line version of the catalogue. We are also grateful to the referee, M.P. Véron-Cetty, for helpful comments.

\section{References}

Angel, J. R. P., \& Stockman, H. S. 1980, ARAA, 18, 321

Bennett, C. L., Hill, R. S., Hinshaw, G., et al. 2003, ApJS, 148, 97

Blandford, R. D., \& Rees, M. J. 1978, in Pittsburgh Conf. on BL Lac Objects, ed. A. N. Wolfe, 328

Burbidge, G., \& Hewitt, A. 1987, AJ, 93, 1

Caccianiga, A., Maccacaro, T., Wolter, A., della Ceca, R., \& Gioia, I. M. 1999, ApJ, 513, 51

Caccianiga, A., Maccacaro, T., Wolter, A., Della Ceca, R., \& Gioia, I. M. 2002a, ApJ, 566, 181

Caccianiga, A., Marchã, M. J., Antòn, S., Mack, K.-H., \& Neeser, M. J. 2002b, MNRAS, 329, 877

Collinge, M. J., Strauss, M. A., Hall, P. B., et al. 2005, AJ, 129, 2542

Condon, J. J., Cotton, W. D., Greisen, E. W., et al. 1998, AJ, 115, 1693

Croom, S. M., Smith, R. J., Boyle, B. J., et al. 2001, MNRAS, 322, L29

Dallacasa, D., Stanghellini, C., Centonza, M., \& Fanti, R. 2000, A\&A, 363, 887

di Serego-Alighieri, S., Danziger, I. J., Morganti, R., \& Tadhunter, C. N. 1994, MNRAS, 269, 998

Giommi, P., \& Colafrancesco, S. 2004, AAP, 414, 7

Giommi, P., Menna, M. T., \& Padovani, P. 1999, MNRAS, 310, 465

Giommi, P., Capalbi, M., et al. 2002, in Blazars Astrophysics with BeppoSAX and Other Observatories, ASI Spec. Publ., 63

Giommi, P., Piranomonte, S., Perri, M., \& Padovani, P. 2005, A\&A, 434, 385

Giommi, P., Capalbi, M., Cavazzuti, E., et al. 2007, A\&A, 468, 571

Hartman, R. C., Bertsch, D. L., Bloom, S. D., et al. 1999, ApJS, 123, 79

Healey, S. E., Romani, R. W., Roger, W., et al. 2007, ApJS, 171, 61

Healey, S. E., Romani, R. W., Cotter, G., et al. 2008, ApJS, 175, 97

Impey, C. D., \& Tapia, S. 1988, ApJ, 333, 666

Jackson, C. A., Wall, J. V., Shaver, P. A., et al. 2002, A\&A, 386, 97

Labiano, A., Barthel, P. D., O’Dea, C. P., et al. 2007, A\&A, 463, 97

Landt, H., Padovani, P., Perlman, E. S., et al. 2001, MNRAS, 323, 757

Laurent-Muehleisen, S. A., Koolgaard, R. I., Ciardullo, R., et al. 1998, ApJS, 118,127

Londish, D., Croom, S. M., Boyle, B. J., et al. 2002, MNRAS, 334, 941

Londish, D., Croom, S. M., Heidt, J., et al. 2007, MNRAS, 374, 556

Mauch, T., Murphy, T., Buttery, H. J., et al. 2003, MNRAS, 342, 1117

Marchã, M. J., Caccianiga, A., Browne, I. W. A., \& Jackson, N. 2001, MNRAS, 326,1455 
Massaro, E., Sclavi, S., et al. 2005, Multifrequency Catalogue of BLAZARS, Volume I (0h-6h) (Roma: Aracne)

Massaro, E., Giommi, P., et al. 2008, Multifrequency Catalogue of BLAZARS,

Volume II (6h-12h) (Roma: Aracne)

Moore, R. L., \& Stockman, H. S. 1981, ApJ, 243, 60

Myers, S. T., Jackson, N. J., Browne, I. W. A., et al. 2003, MNRAS, 341, 1

Nesci, R., Sclavi, S., \& Massaro, E. 2005, A\&A, 434, 895

O’Dea, C. P., Baum, S. A., \& Stanghellini, C. 1991, ApJ, 380, 66

The Pierre Auger Collaboration 2007, Science, 318, 939

Padovani, P., \& Giommi, P. 1995, MNRAS, 277, 147

Perlman, E. S., Stocke, J. T., Schachter, J. F., et al. 1996, ApJS, 104, 251

Perlman, E. S., Padovani, P., Giommi, P., et al. 1998, AJ, 115, 1253

Plotkin, R. M., Anderson, S. F., Patrick, B., et al. 2008, AJ, 135, 2453

Rector, T. A., Stocke, J. T., Perlman, E. S., Morris, S. L., \& Gioia, I. M. 2000, AJ, 120, 1626

Sowards-Emmerd, D., Romani, R. W., Michelson, P. F., Healey, S. E., \& Nolan, P. L. 2005, ApJ, 626, 95

Stein, W. A., O’Dell, S. L., \& Strittmatter, P. A. 1976, ARAA, 14, 173
Stickel, M., Fried, J. W., Kuehr, H., Padovani, P., \& Urry, C. M. 1991, ApJ, 374, 431

Stocke, J. T., Morris, S. L., Gioia, I. M., et al. 1991, ApJS, 76, 813

Strittmatter, P. A., Serkowski, K., Carswell, R., et al. 1972, ApJ, 175, L7

Taker, N. 2000, Ph.D. Thesis, Macquarie University

Torniainen, I., Tornikoski, M., Teräsranta, H., Aller, M. F., \& Aller, H. D. 2005 , A\&A, 435, 839

Turriziani, S., Cavazzuti, E., \& Giommi, P. 2007, A\&A, 472, 699

Urry, M. C., \& Padovani, P. 1995, PASP, 107, 803

Voges, W., Aschenbach, B., Boller, Th., et al. 1999, A\&A, 349, 389

Véron-Cetty, M.-P., \& Véron, P. 2006, A\&A, 455, 773

Wall, J. V., \& Peacock, J. A. 1985, MNRAS, 216, 173

Wall, J. V., Jackson, C. A., Shaver, P. A., Hook, I. M., \& Kellermann, K. I. 2005, A\&A, 434, 133

Wei, C. 2006, Proc. Vulcano Worshop 2006, in press [arxiv: astro-ph/0608042]

White, R. L., Becker, R. H., et al. 1997, ApJ, 475, 479 